\title{
Controlling Multiple Fluorescent Signal Output in Cyclic peptide-Based
}

Supramolecular Systems

Roberto J. Brea, M. Eugenio Vázquez, Manuel Mosquera ${ }^{\dagger}$, Luis Castedo, Juan R. Granja*

[*] R. J. Brea, Dr. M. E. Vázquez, Prof. L. Castedo, Prof. J. R. Granja Departamento de Química Orgánica, Facultade de Quimica, Universidade de Santiago de Compostela, 15782 Santiago de Compostela, SPAIN.

qojuangg@usc.es

[†] Prof. M. Mosquera

Departamento de Química Física, Facultade de Química, Universidade de Santiago de Compostela, 15782 Santiago de Compostela, SPAIN.

SUPPORTING INFORMATION 

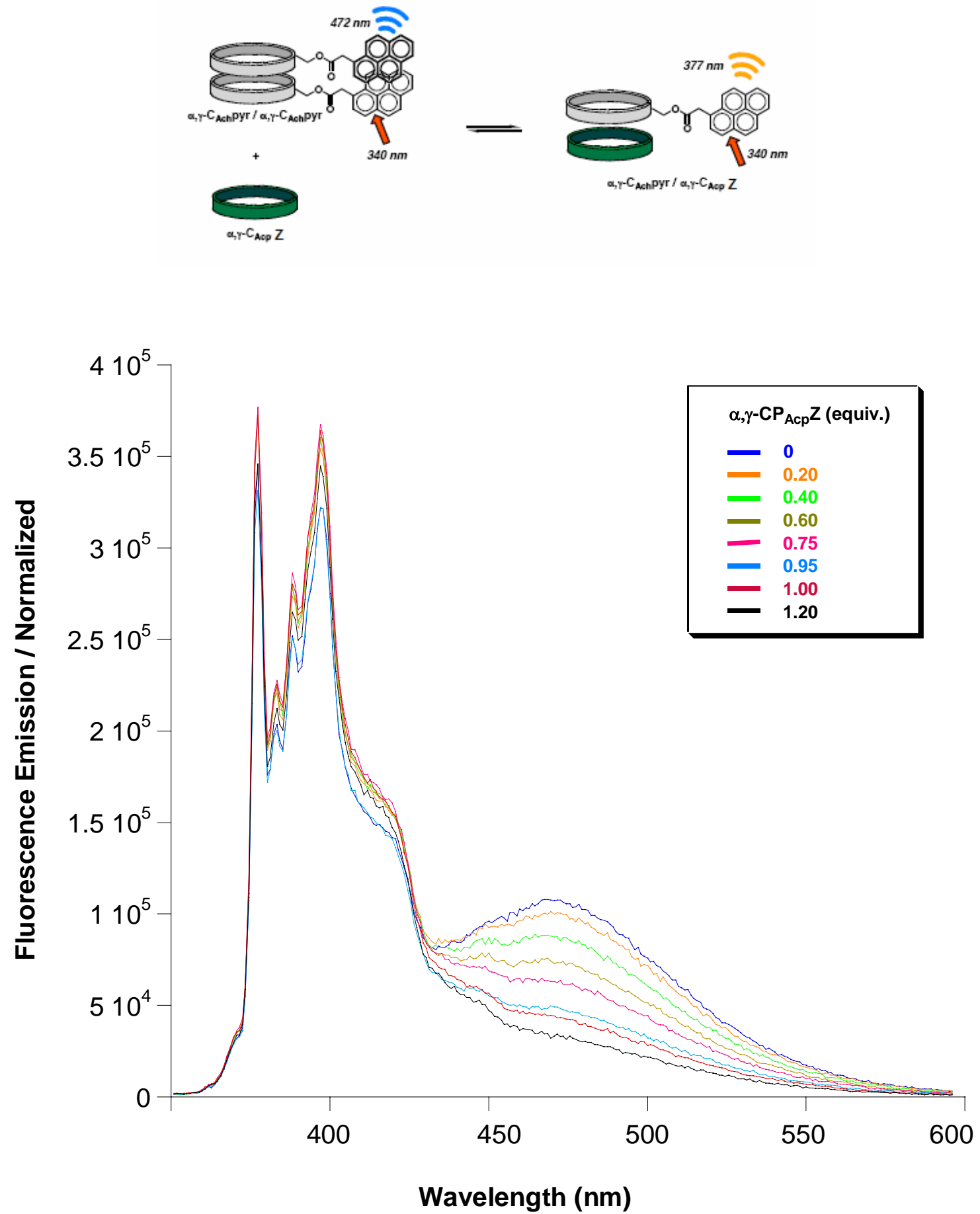

Figure S1. Fluorescence emission spectra of a $3.68 \mu \mathrm{M} \alpha, \gamma-\mathbf{C P}_{\text {Ach }} \mathbf{p y r}$ solution in the presence of different equivalents (from 0 to 1.2 equiv.) of $\alpha, \gamma-\mathbf{C P}_{\text {Acp }} \mathbf{Z}$ [All spectra in $\mathrm{CHCl}_{3}$. Excitation wavelength was $340 \mathrm{~nm}$. Spectra were normalized for clear representation]. 

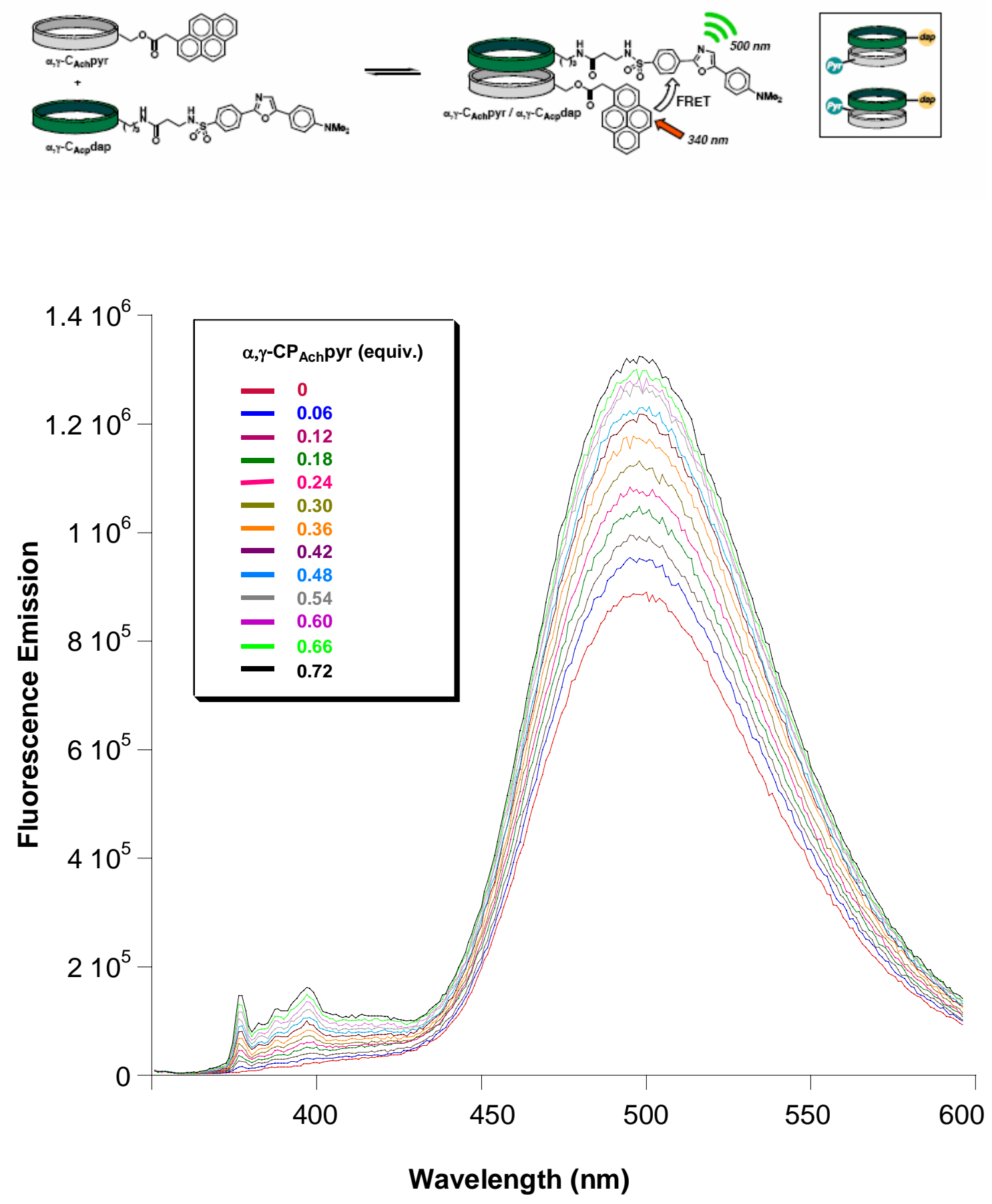

Figure S2. Fluorescence emission spectra of a $0.81 \mu \mathrm{M} \alpha, \gamma-\mathbf{C P}_{\text {Acp }} \mathbf{d a p}$ solution in the presence of different equivalents (from 0 to 0.72 equiv.) of $\alpha, \gamma-\mathbf{C P}_{\text {Ach }} \mathbf{p y r}$ [All spectra in $\mathrm{CHCl}_{3}$. Excitation wavelength was $340 \mathrm{~nm}$ ]. 


\section{* General Methods, Instrument Details and Materials}

-General. Commercially available N-Boc amino acids, O-(7-azabenzotriazol-1-yl)-1,1,3,3tetramethyluronium hexafluorophosphate (HATU) and $O$-Benzotriazol-1-yl- $N, N, N^{\prime}, N^{\prime}-$ tetramethyluronium tetrafluoroborate (TBTU) were all used as obtained from Novabiochem, Applied Biosystems or Bachem. Deuterated chloroform $\left(\mathrm{CDCl}_{3}\right)$, 1-Pyreneacetic acid, $N, N^{\prime}$ Diisopropylcarbodiimide (DIC) and 4-Dimethylaminopyridine (DMAP) were obtained from Aldrich. Dapoxil ${ }^{\circledR}$ 3-sulfonamido propionic acid succinimidyl ester was obtained from Molecular Probes. All other reagents obtained from commercial suppliers were used without further purification unless otherwise noted. Dichloromethane (DCM) and piperidine were dried and distilled over calcium hydride. ${ }^{\mathbf{1 , 2}}$ DIEA was dried and distilled over calcium hydride, and then redistilled over nynhidrin ${ }^{\mathbf{1 2}}$. DMF used in coupling steps was dried over $4 \AA$ molecular sieves. Analytical thin-layer chromatography was performed on E. Merck silica gel $60 \mathrm{~F}_{254}$ plates. Compounds, which were not UV active, were visualized by dipping the plates in a nynhidrin solution and heating. Silica gel flash chromatography was performed using E. Merck silica gel (type 60SDS, 230-400 mesh). Preparative thin-layer chromatography was performed on E. Merck silica gel $60 \mathrm{~F}_{254}$ plates $(1 \mathrm{~mm})$. Solvent mixtures for chromatography are reported as v/v ratios. HPLC purification was carried out on Phenomenex Maxsil-10 silica column with $\mathrm{CH}_{2} \mathrm{Cl}_{2} / \mathrm{MeOH}$ gradients between 100 and 85:15. Proton nuclear magnetic resonance ( ${ }^{1} \mathrm{H}$ NMR) spectra were recorded on Varian Inova-750 MHz, Bruker AMX-500 MHz or Bruker WM-250 MHz spectrometers. Chemical shifts were reported in parts per million (ppm, $\delta$ ) relative to tetramethylsilane $(\delta 0.00)$. ${ }^{1} \mathrm{H}$ NMR splitting patterns are designated as singlet (s), doublet (d), triplet (t), quartet (q) or pentuplet (p). All first-order splitting patterns were assigned on the basis of the appearance of the multiplet. Splitting patterns that could not be easily interpreted are designated as multiplet (m) or broad (br). Carbon nuclear magnetic resonance $\left({ }^{13} \mathrm{C} \mathrm{NMR}\right)$ spectra were recorded on Varian Mercury-300 MHz, Bruker WM-250 $\mathrm{MHz}$ or Bruker AMX-500 MHz spectrometers. Carbon resonances were assigned using distortionless enhancement by polarization transfer (DEPT) spectra obtained with phase angles of 135. Fast Atom Bombardement (FAB) mass spectra were recorded on a Micromass Autospec mass spectrometer. Mass Spectrometry of Laser Desorption/Ionization-Time of Flight (MALDI-TOF) was obtained on a Bruker Autoflex mass spectrometer. UV/Vis measurements were made on a Cary 100 Bio UV/Visible spectrophotometer, using $\mathrm{NaCl}$ solution UV cells. FTIR measurements were made on a JASCO FT/IR400 spectrophotometer using $5-10 \mathrm{mM}$ in $\mathrm{CHCl}_{3}$ and placed in a $\mathrm{NaCl}$ solution IR cell. Fluorescence measurements were obtained on a SPEX FluoroMax-3 spectrofluorometer. Fluorescence lifetime measurements were made on an Edinburgh Instruments CD900 Single Photon Counting (SPC) apparatus.

- ${ }^{1}$ H-NMR Assignments of Cyclic Peptides. The signals of the ${ }^{1} \mathrm{H}$ NMR spectra of the peptides in $\mathrm{CDCl}_{3}$ were identified from the corresponding double-quantum-filled 2D COSY (2QF-COSY), TOCSY and/or NOESY and ROESY spectra acquired at concentration and temperature indicated. Mixing times

\footnotetext{
${ }^{1}$ Brown, H. C. “Organic Synthesis via Boranes”, Ed. John Wiley \& Sons, 1975

${ }^{2}$ Perrin, D. D.; Armarego, W. I. F. "Purification of Laboratory Chemicals”, Ed. Pergamon Press, 1988
} 
( 250 ms or $400 \mathrm{~ms}$ ) were not optimized. Spectra were typically acquired using Bruker standard pulse sequences on $500 \mathrm{MHz}$ apparatuses, and were referenced relative to residual proton resonances in $\mathrm{CDCl}_{3}$ (at $7.26 \mathrm{ppm}$ ). ${ }^{1} \mathrm{H}-\mathrm{NMR}$ spectra also were obtained on a Varian Inova-750 MHz spectometer. 


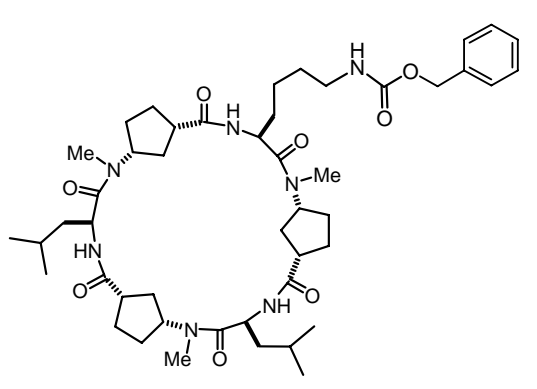

cyclo $\left\{\left[L-\text { Leu- } D-{ }^{\mathrm{Me}} \mathrm{N}-\gamma-\mathrm{Acp}\right]_{2}-\mathrm{L}-\mathrm{Lys}(\mathrm{Z})-D_{-}{ }^{\mathrm{Me}} \mathrm{N}-\gamma-\mathrm{Acp}-\right\}\left(\alpha, \gamma-\mathrm{CP}_{\mathrm{Acp}} \mathrm{Z}\right)$
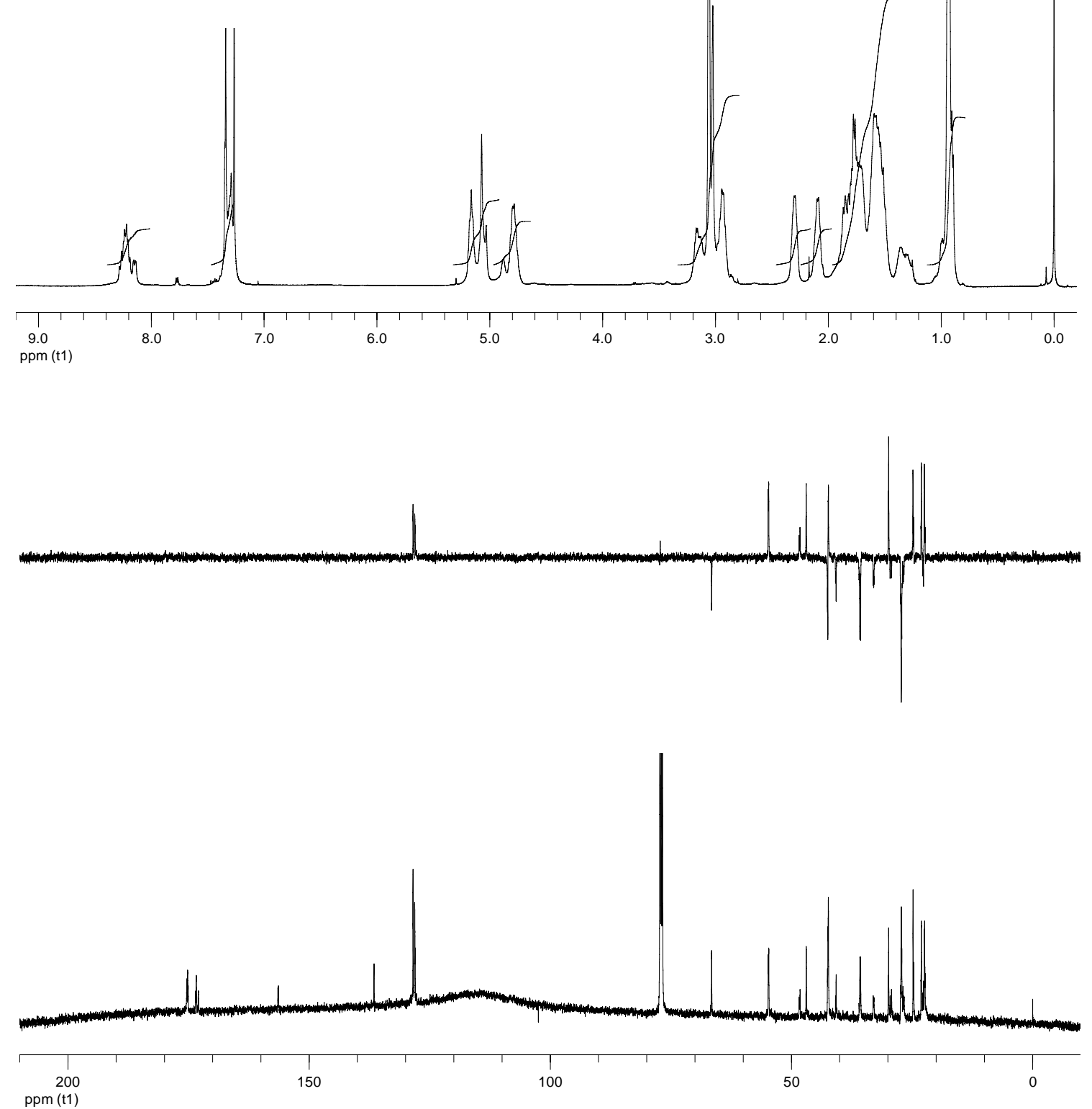
cyclo $\left\{\left[L-\text { Leu- } D-{ }^{\mathrm{Me}} \mathrm{N}-\gamma-\mathrm{Acp}\right]_{2}-L-\operatorname{Lys}(\mathrm{Z})-D_{-}{ }^{\mathrm{Me}} \mathrm{N}-\gamma-\mathrm{Acp}-\right\}\left(\alpha, \gamma-\mathrm{CP}_{\mathrm{Acp}} \mathrm{Z}\right)$

1) $\operatorname{COSY}\left[34.76 \mathrm{mM}, \mathrm{CDCl}_{3}, 298 \mathrm{~K}\left(25^{\circ} \mathrm{C}\right), 500.13 \mathrm{MHz}\right]$

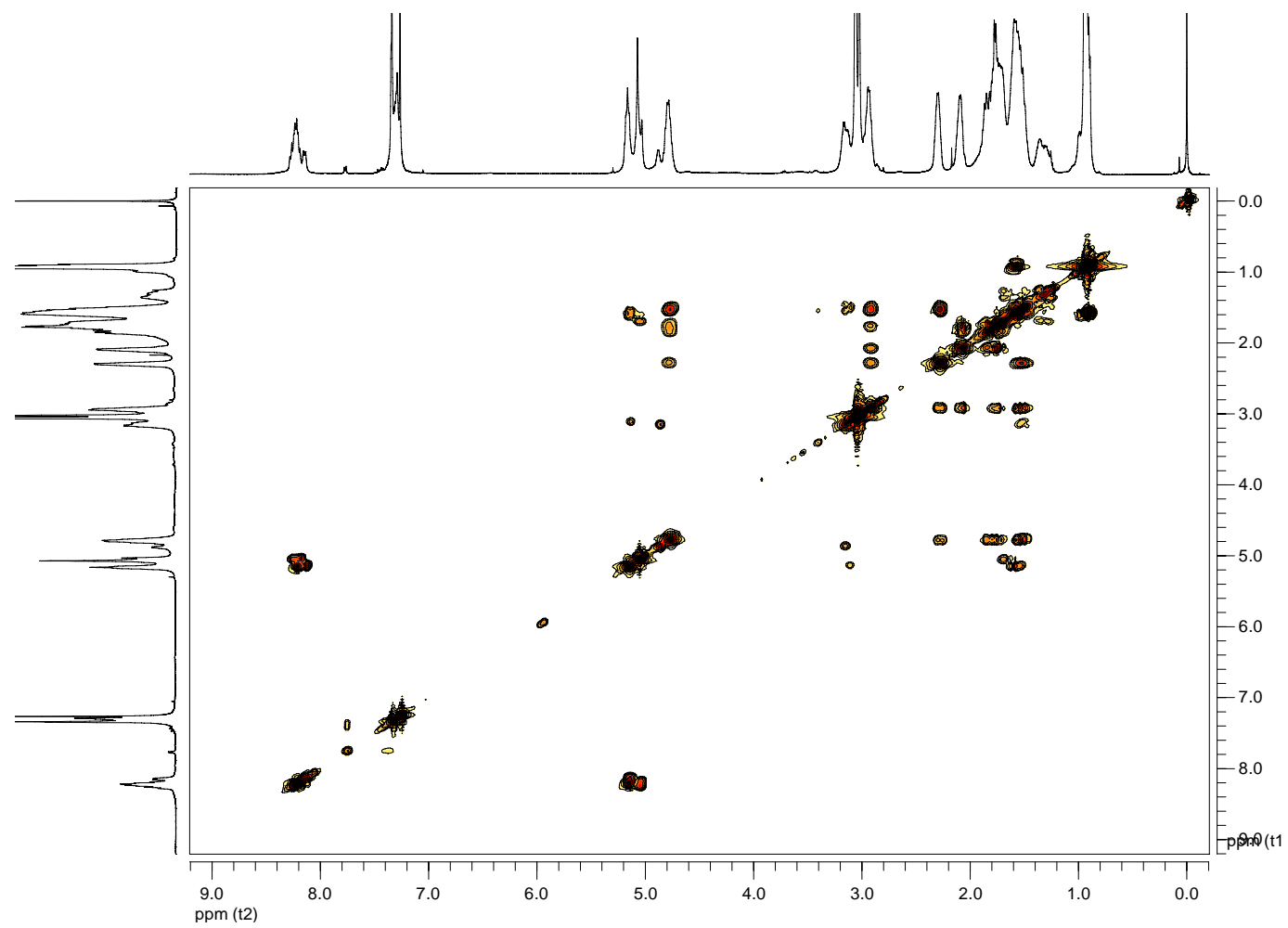

2) ROESY [34.76 mM, $\left.\mathrm{CDCl}_{3}, 298 \mathrm{~K}\left(25^{\circ} \mathrm{C}\right), 500.13 \mathrm{MHz}\right]$

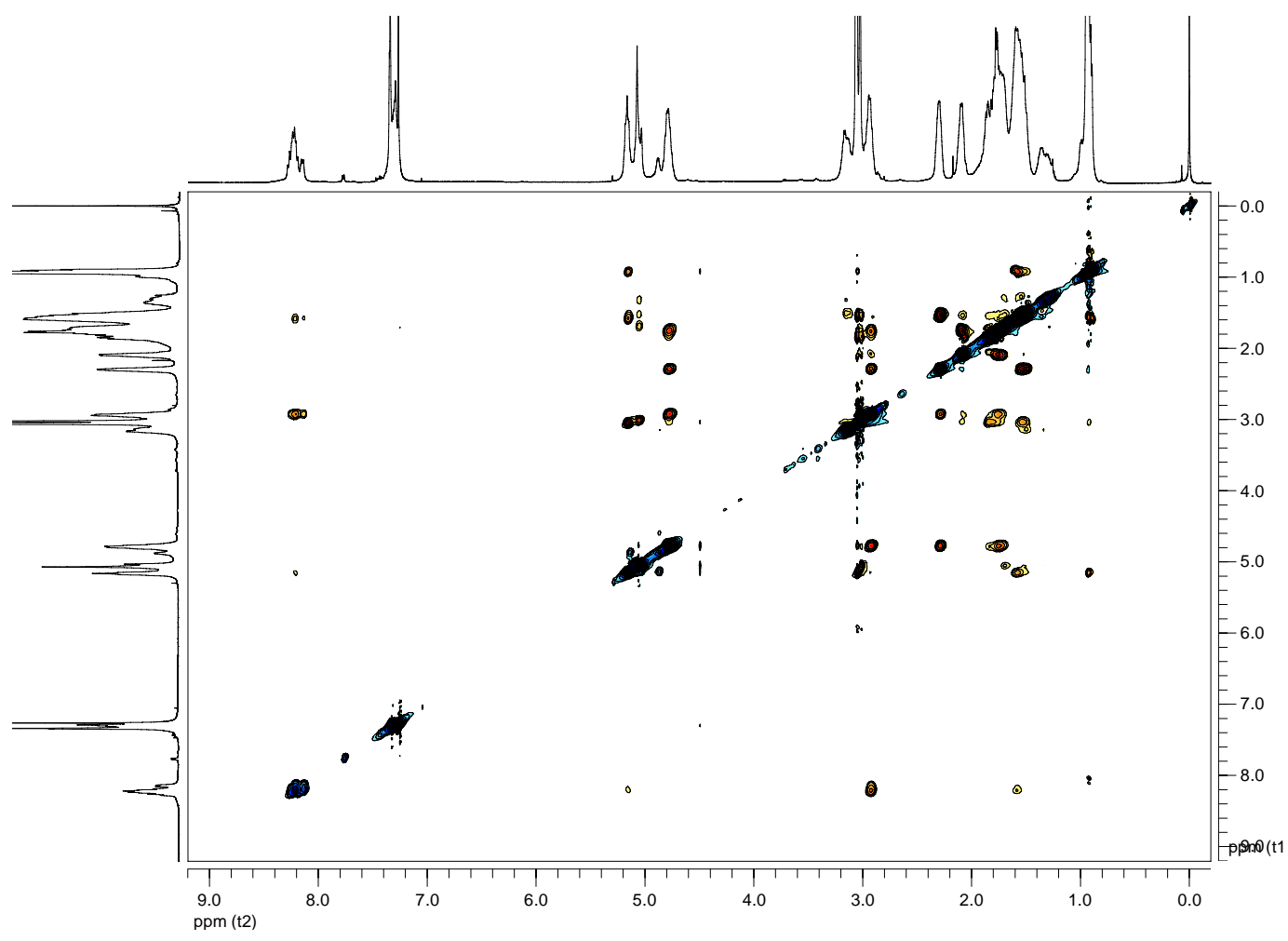


3) NOESY [34.76 mM, $\left.\mathrm{CDCl}_{3}, 298 \mathrm{~K}\left(25^{\circ} \mathrm{C}\right), 500.13 \mathrm{MHz}\right]$

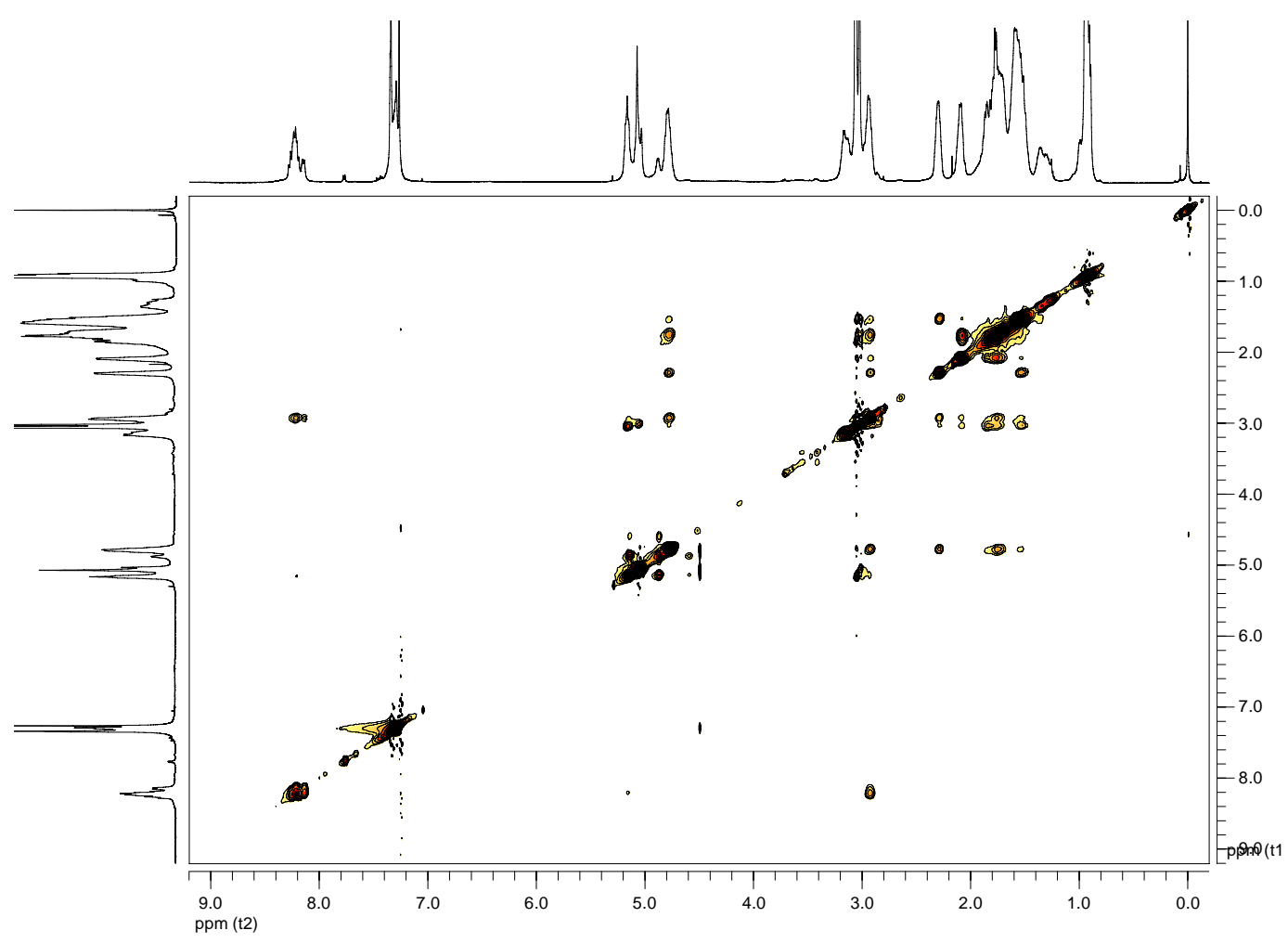

4) TOCSY [34.76 mM, $\left.\mathrm{CDCl}_{3}, 298 \mathrm{~K}\left(25^{\circ} \mathrm{C}\right), 500.13 \mathrm{MHz}\right]$

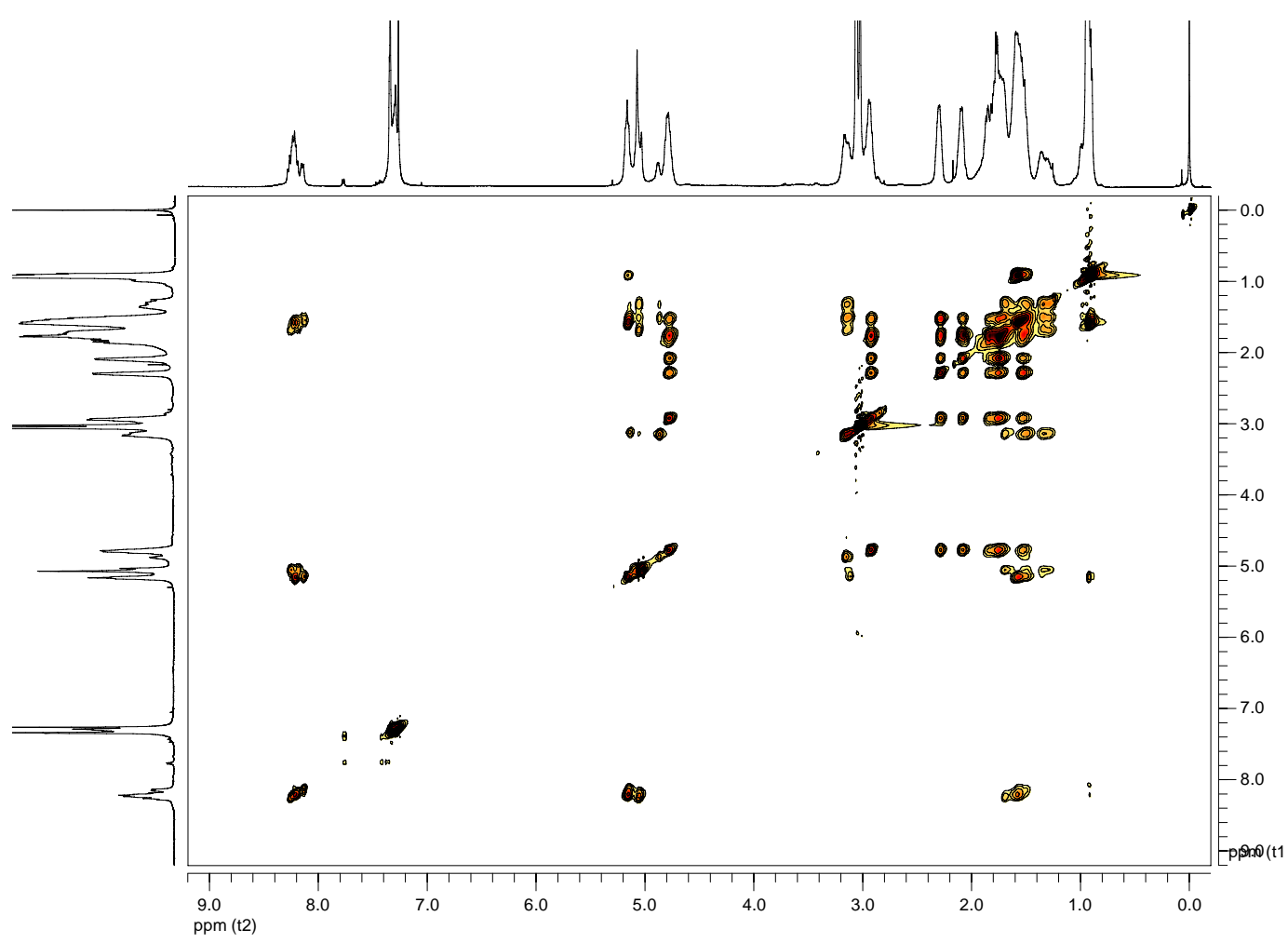


5) FT-IR $\left[\mathrm{CHCl}_{3}, 298 \mathrm{~K}\left(25^{\circ} \mathrm{C}\right)\right]$

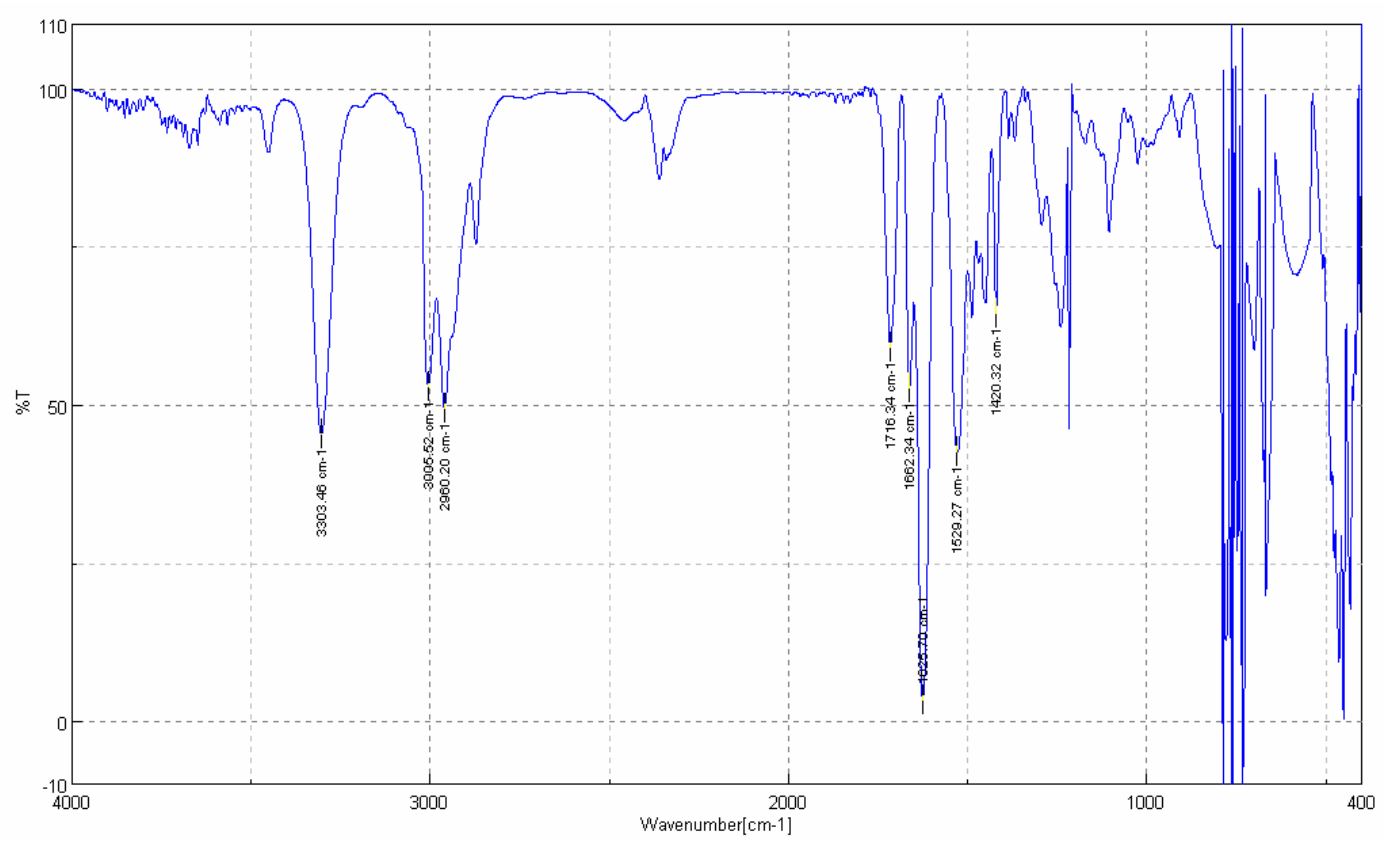




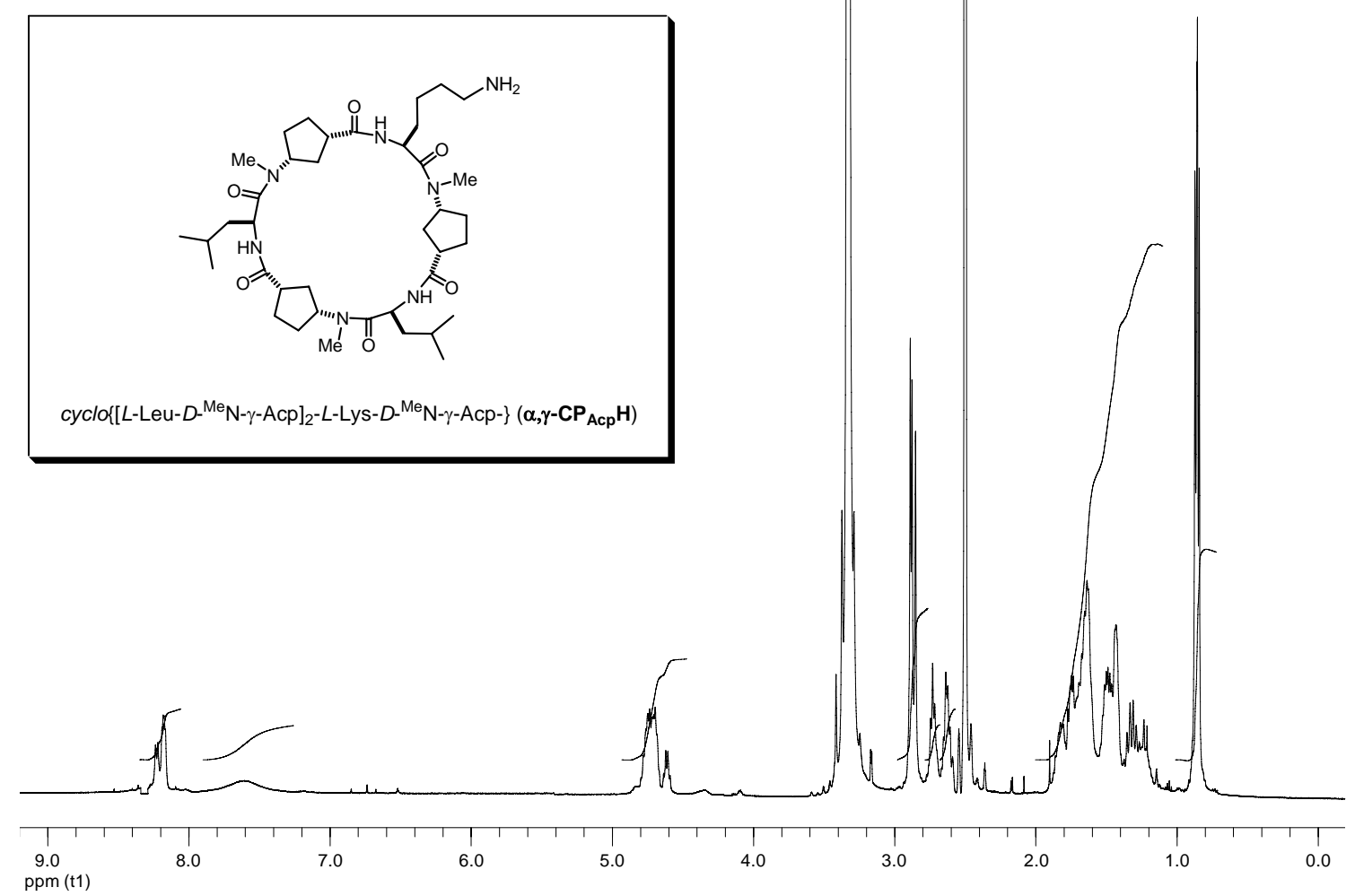



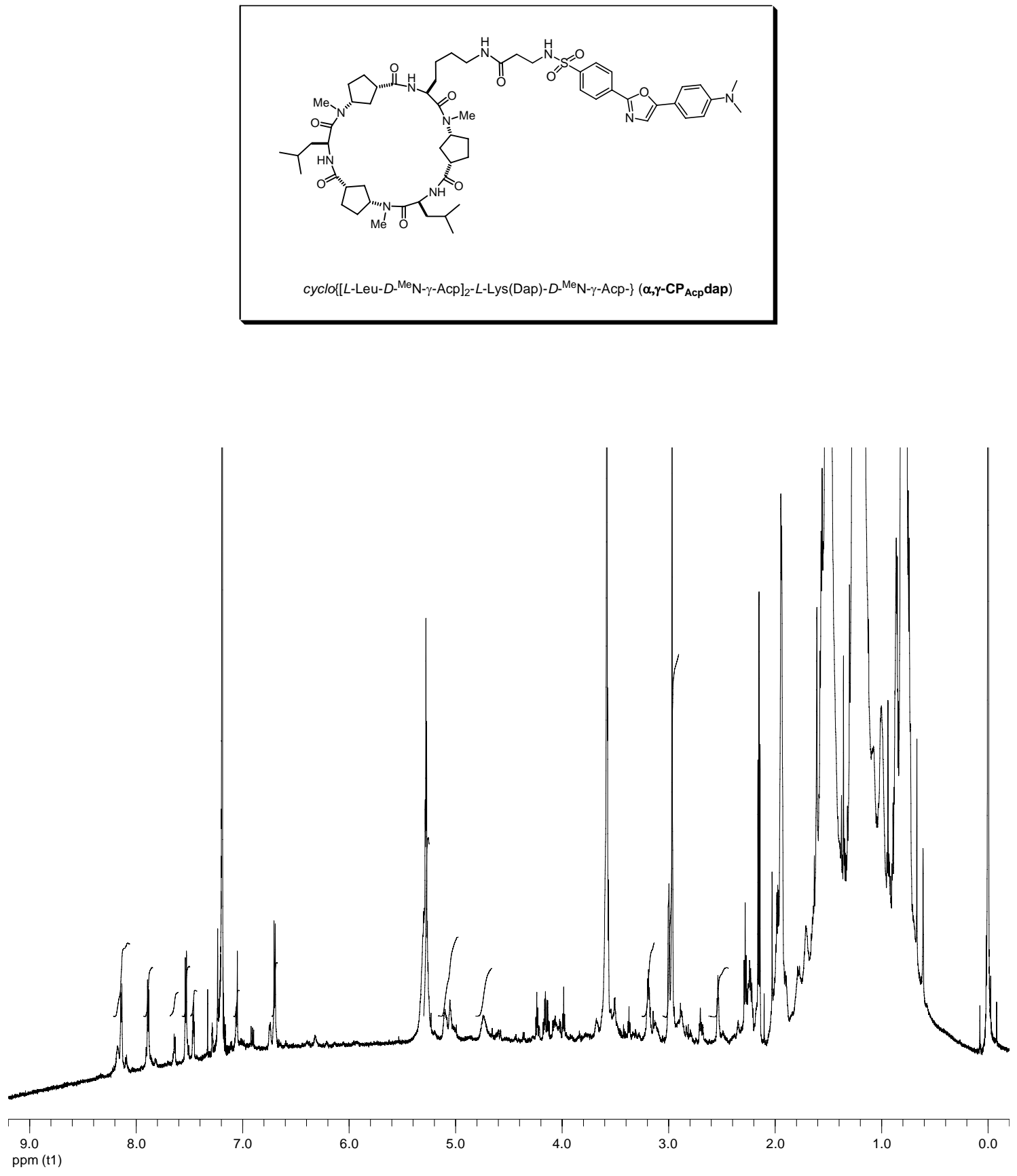
cyclo $\left\{\left[L-\text { Leu- } D-{ }^{\mathrm{Me}} \mathrm{N}-\gamma-\mathrm{Acp}\right]_{2}-L-\mathrm{Lys}(\mathrm{Dap})-D-{ }^{\mathrm{Me}} \mathrm{N}-\gamma-\mathrm{Acp}-\right\}\left(\alpha, \gamma-\mathrm{CP}_{\mathrm{Acp}} \mathrm{dap}\right)$

1) $\operatorname{COSY}\left[5.51 \mathrm{mM}, \mathrm{CDCl}_{3}, 298 \mathrm{~K}\left(25^{\circ} \mathrm{C}\right), 750 \mathrm{MHz}\right]$

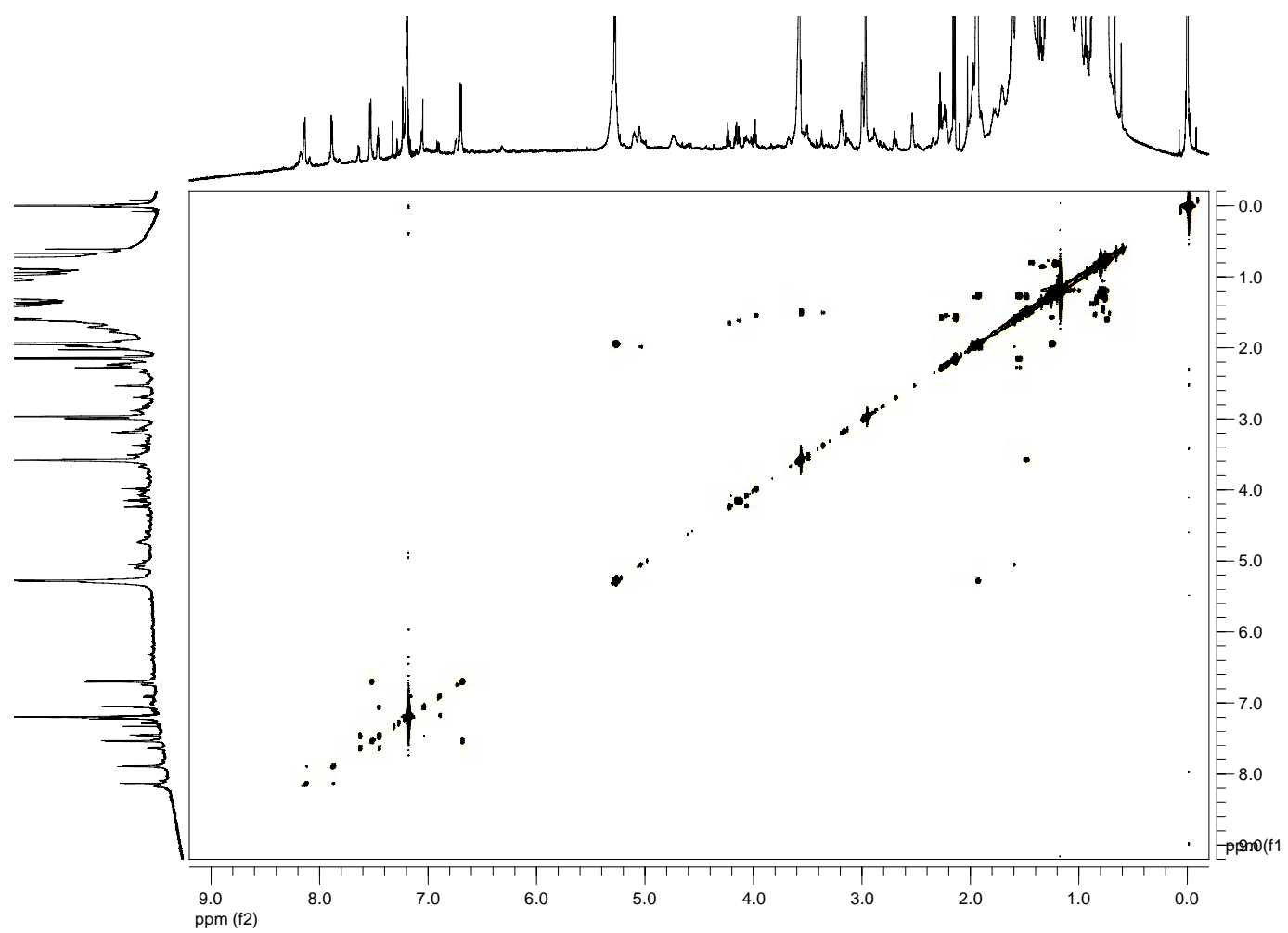

2) ROESY [5.51 mM, $\left.\mathrm{CDCl}_{3}, 298 \mathrm{~K}\left(25^{\circ} \mathrm{C}\right), 750 \mathrm{MHz}\right]$

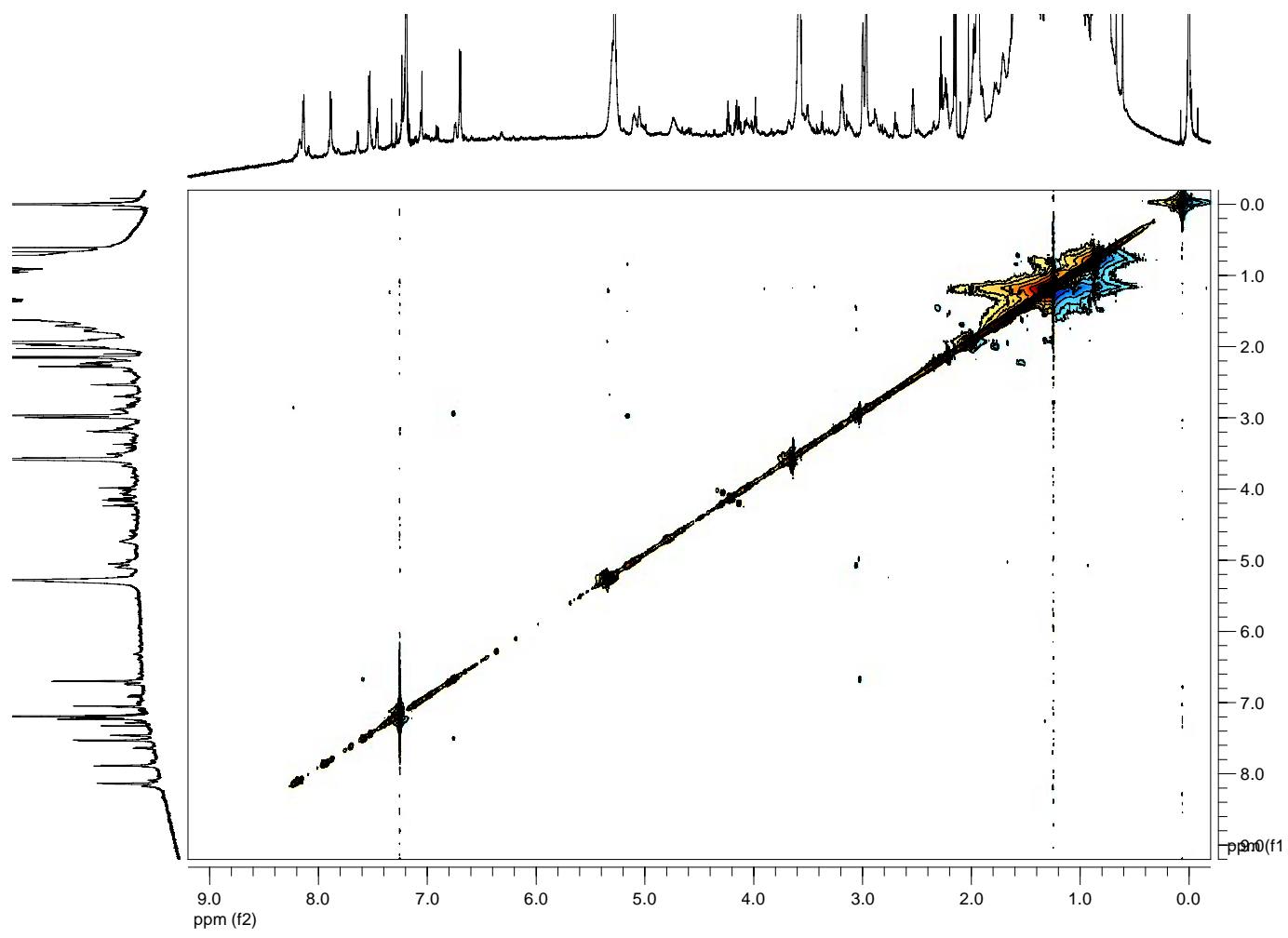


3) NOESY [5.51 mM, CDCl 3 , $\left.298 \mathrm{~K}\left(25^{\circ} \mathrm{C}\right), 750 \mathrm{MHz}\right]$

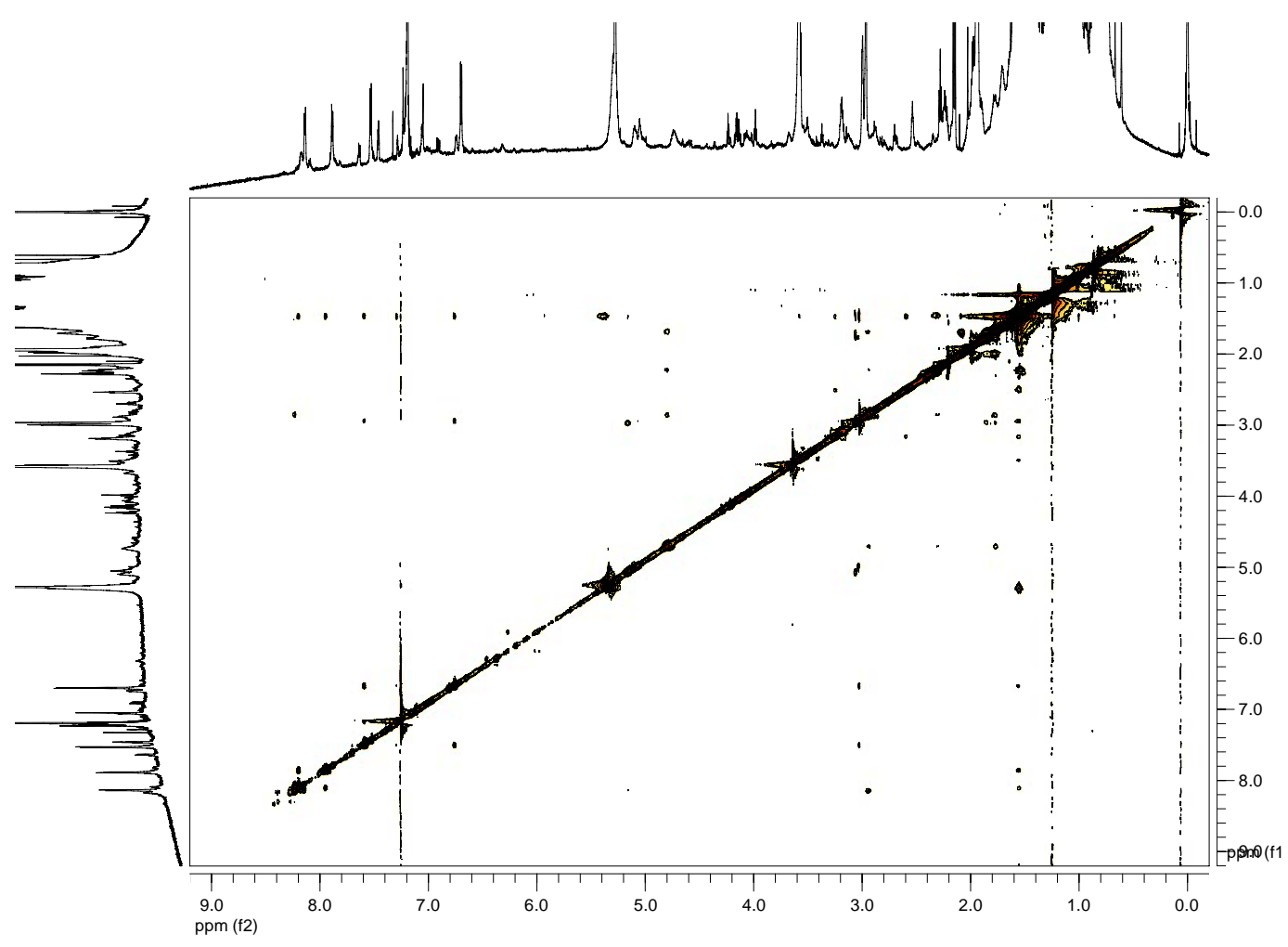

4) TOCSY [5.51 mM, CDCl 3 , $\left.298 \mathrm{~K}\left(25^{\circ} \mathrm{C}\right), 750 \mathrm{MHz}\right]$

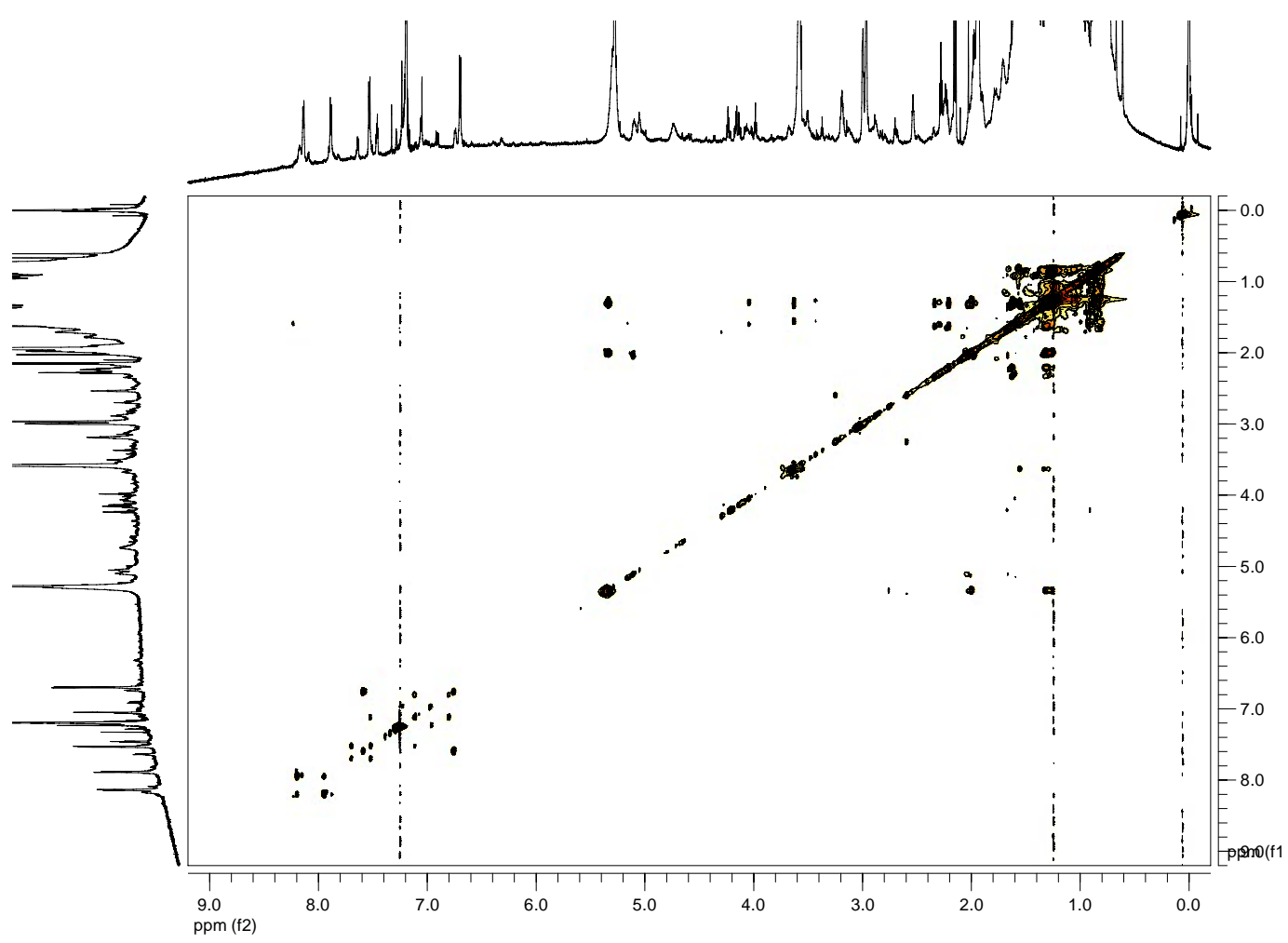




\section{$\underline{\alpha, y-\mathbf{C P}_{\text {Acp }} \text {-dap }}$}

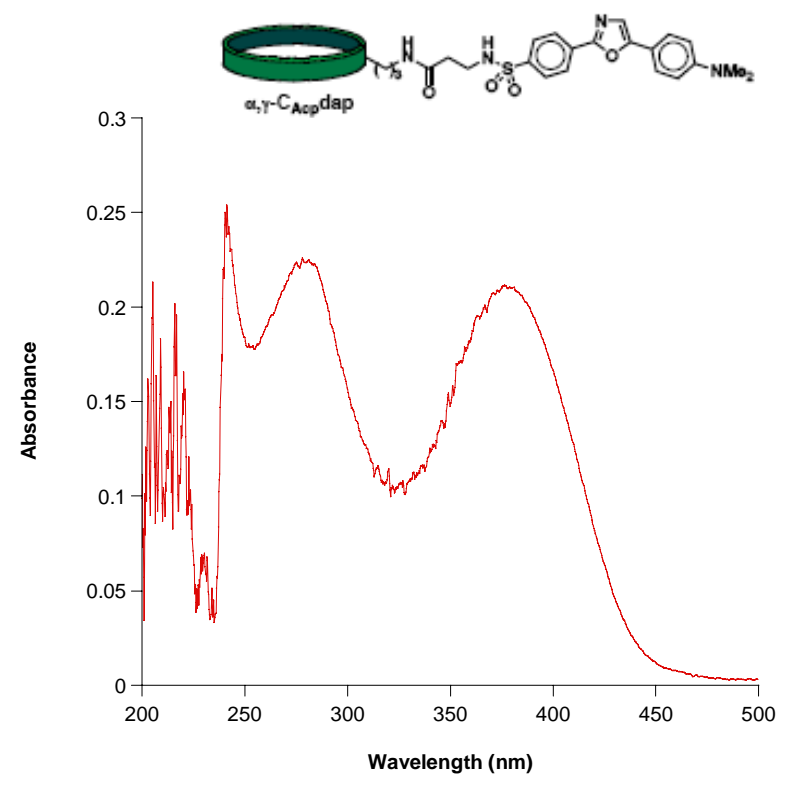

Absorption spectrum of a $7.44 \mu \mathrm{M} \alpha, \gamma-\mathbf{C P}_{\text {Acp }} \mathbf{d a p}$ solution. 

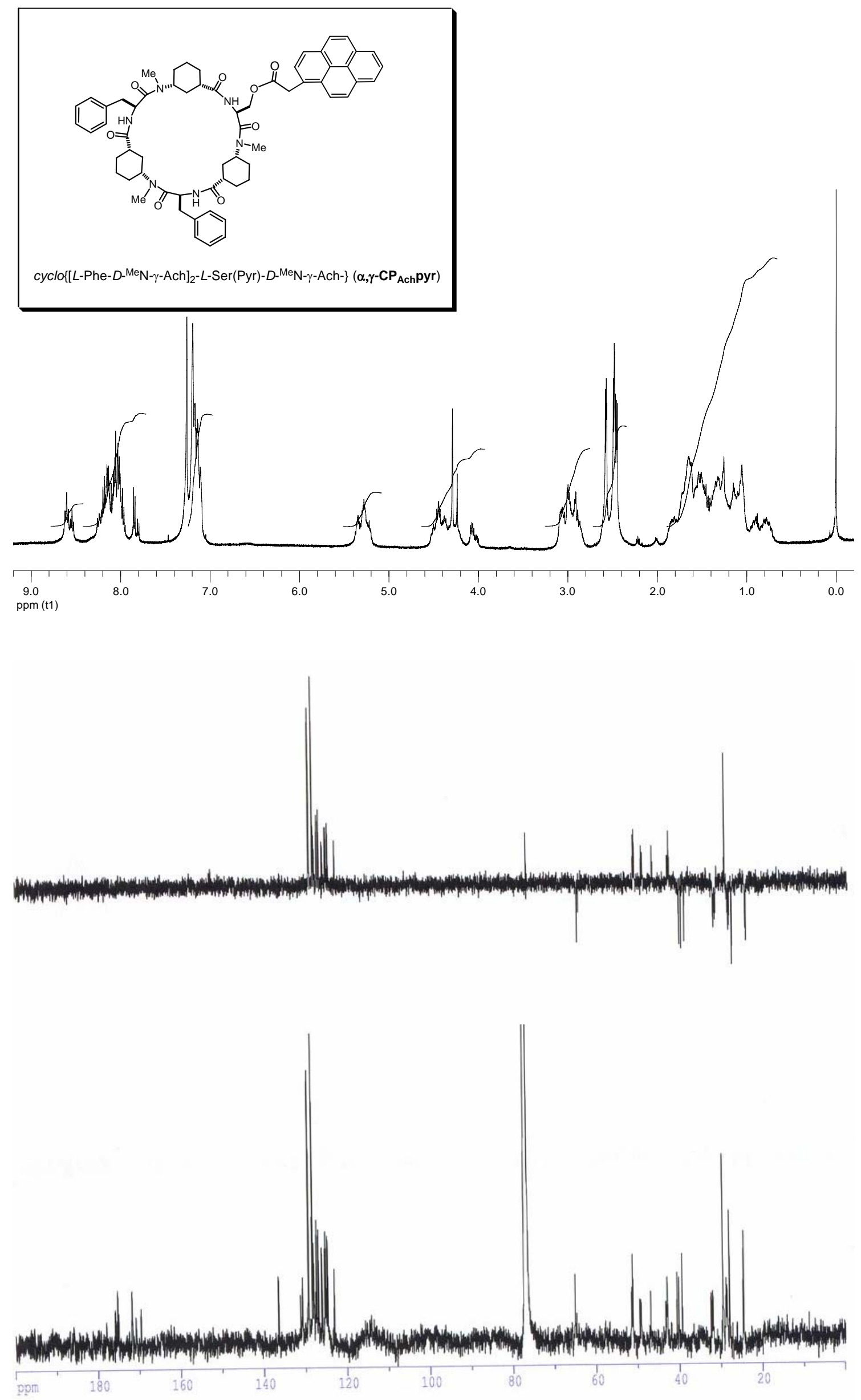
cyclo $\left\{\left[L-\mathrm{Phe}-D-{ }^{\mathrm{Me}} \mathrm{N}-\gamma-\mathrm{Ach}\right]_{2}-L-\mathrm{Ser}(\mathrm{Pyr})-D-{ }^{\mathrm{Me}} \mathrm{N}-\gamma-\mathrm{Ach}-\right\}\left(\alpha, \gamma-\mathrm{CP}_{\mathrm{Ach}} \mathrm{Pyr}\right)$

1) $\operatorname{COSY}\left[10.00 \mathrm{mM}, \mathrm{CDCl}_{3}, 298 \mathrm{~K}\left(25^{\circ} \mathrm{C}\right), 500.13 \mathrm{MHz}\right]$

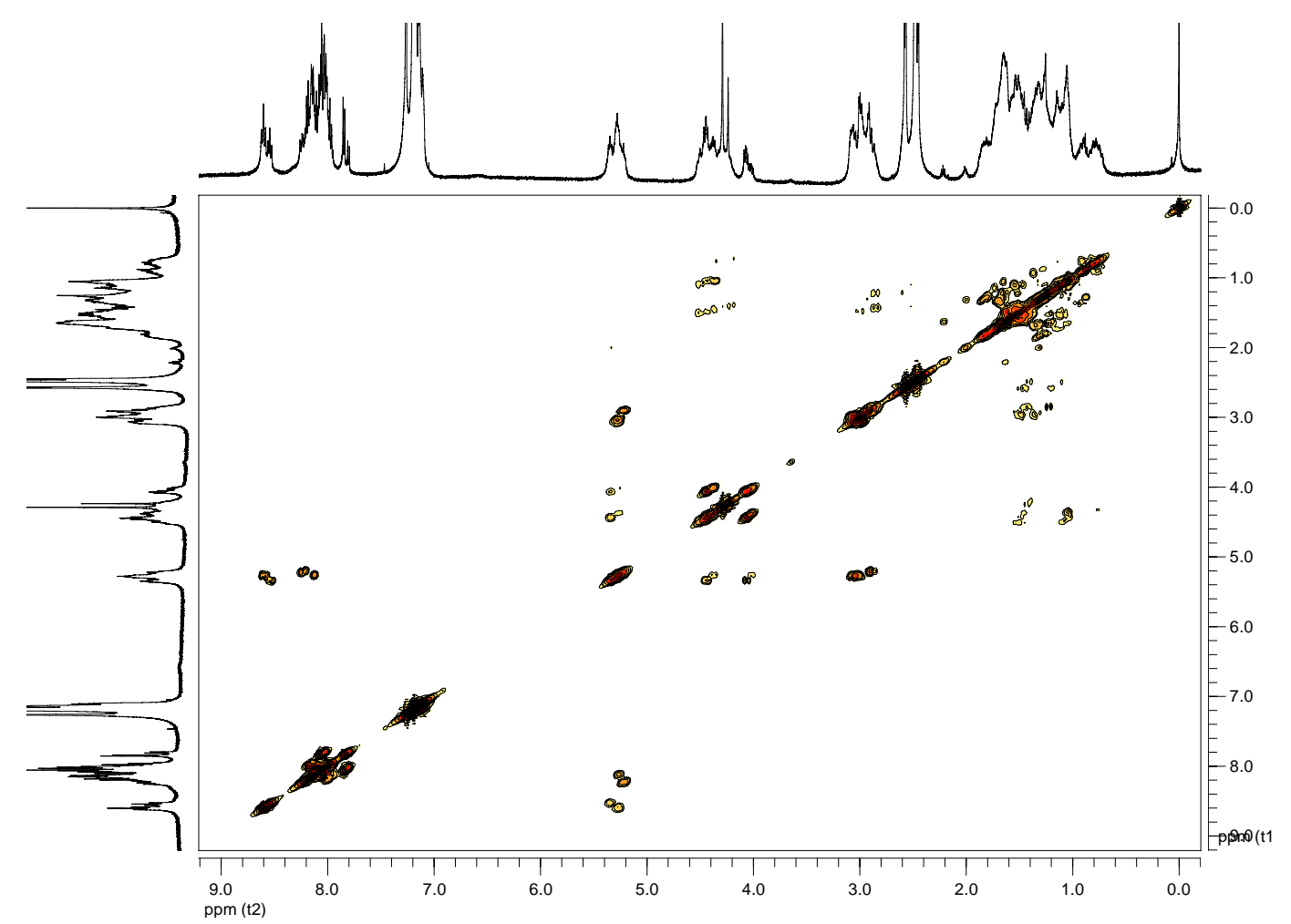

2) ROESY [10.00 mM, $\left.\mathrm{CDCl}_{3}, 298 \mathrm{~K}\left(25^{\circ} \mathrm{C}\right), 500.13 \mathrm{MHz}\right]$

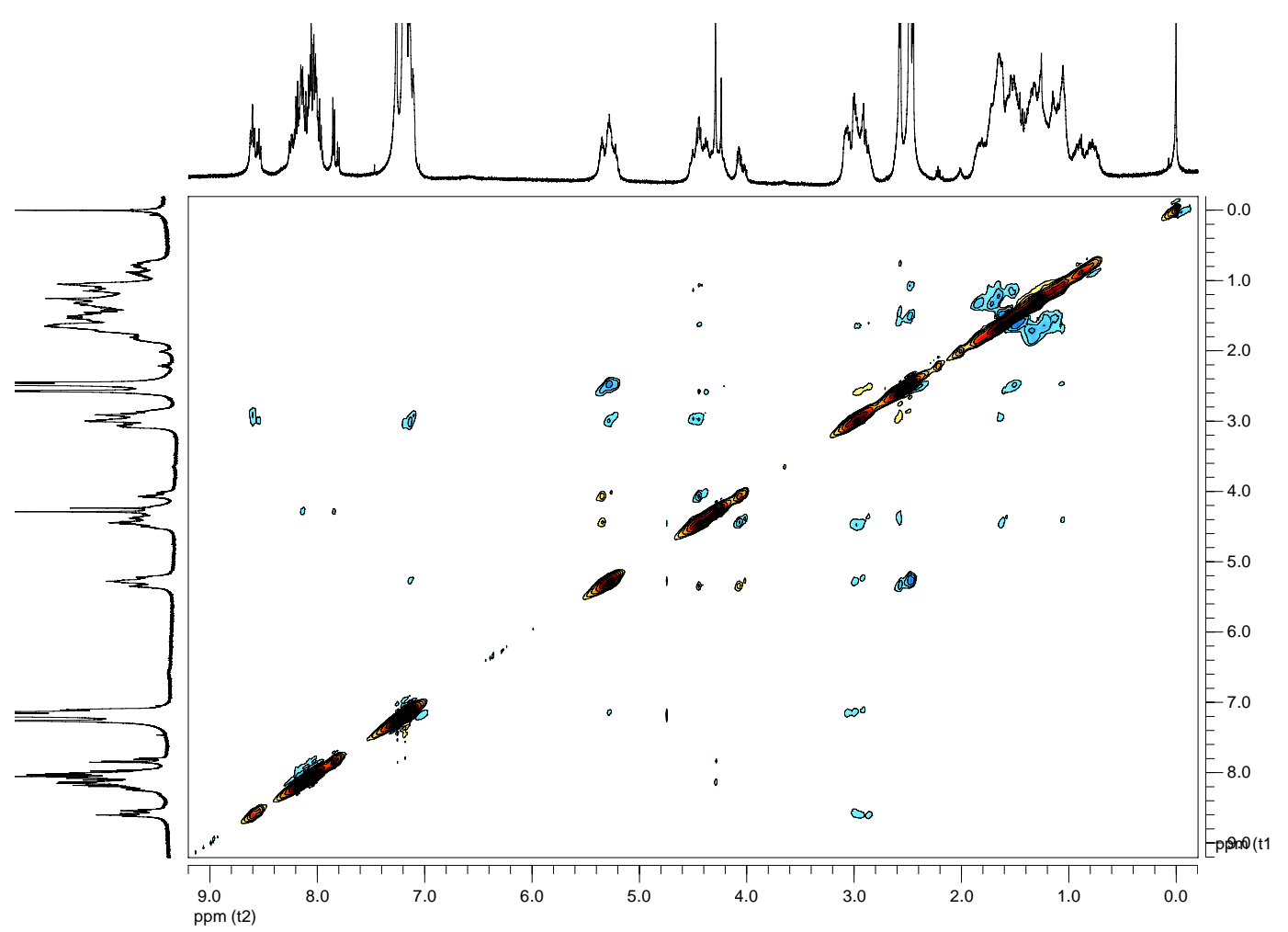


3) NOESY [10.00 mM, CDCl 3 , $\left.298 \mathrm{~K}\left(25^{\circ} \mathrm{C}\right), 500.13 \mathrm{MHz}\right]$

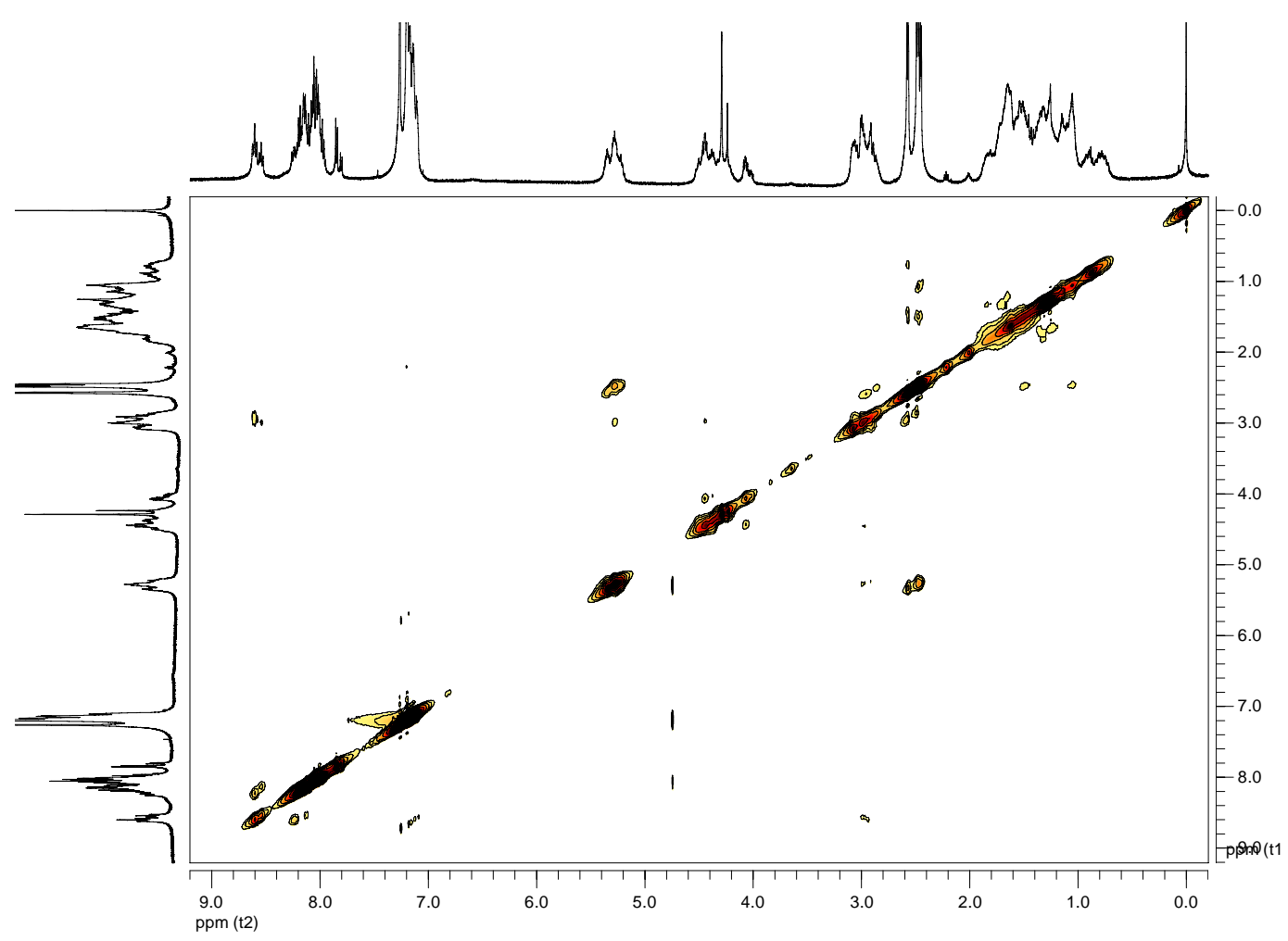

4) TOCSY [10.00 mM, CDCl $\left.3,298 \mathrm{~K}\left(25^{\circ} \mathrm{C}\right), 500.13 \mathrm{MHz}\right]$

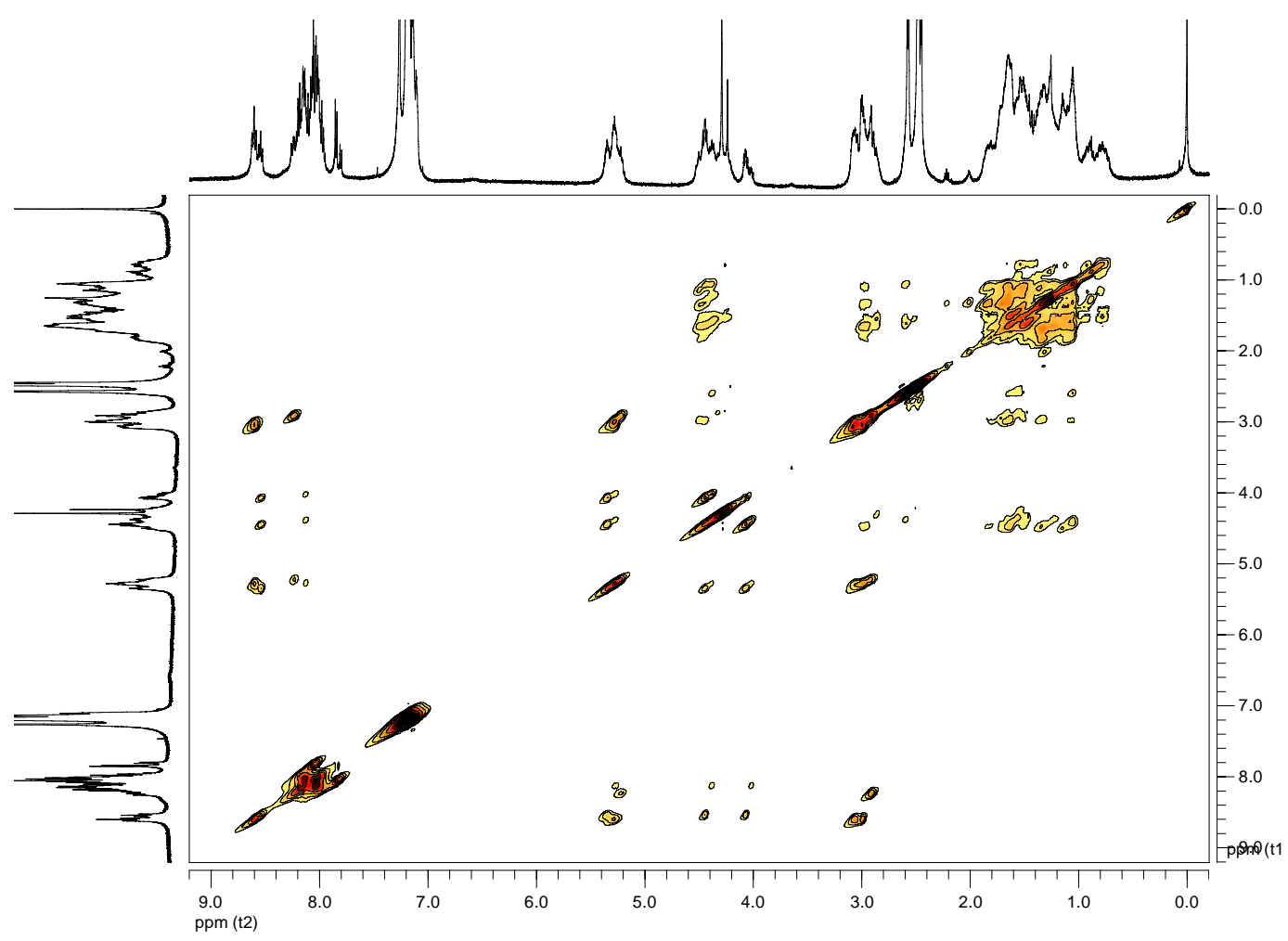




\section{$\underline{\alpha, \gamma-\mathbf{C P}} \underline{\text { Ach }}_{\text {-pyr }}$}

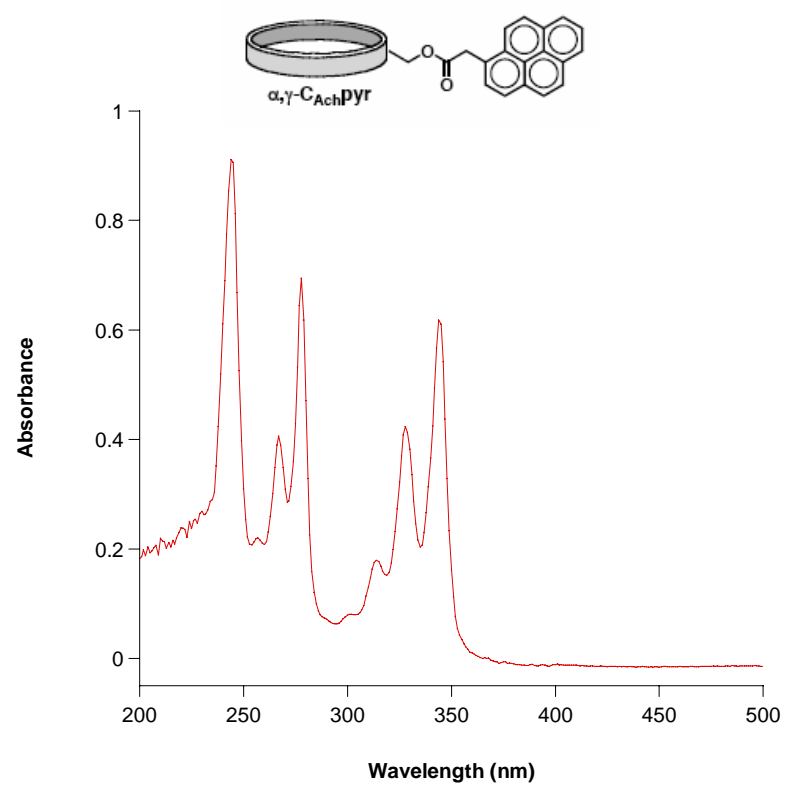

Absorption spectrum of a $13.81 \mu \mathrm{M} \alpha, \gamma-\mathbf{C P} \mathbf{P}_{\text {Ach }}$ pyr solution. 